\title{
Identification of Candida albicans and C. tropicalis with an umbelliferyl-labelled galactosaminide
}

\author{
MUKOMA F. SIMPANYA* \\ Prince Leopold Institute of Tropical Medicine, Mycology Laboratory, Nationalestraat 155, B-2000 Antwerp, Belgium
}

\begin{abstract}
Summary. The 4-methylumbelliferyl-N-acetyl- $\beta$-D-galactosaminide (UAG) test was evaluated in parallel with chlamydospore (CHL) and germ-tube (GT) tests for the identification of Candida spp. The UAG test gave $86.6 \%$ correct identification of $C$. albicans and $C$. tropicalis. Non-C. albicans and non- $C$. tropicalis yeasts gave correct negative results in UAG tests. Only one isolate of Trichosporon beigelii gave a "false" positive reaction. The UAG test, which can be completed within $30 \mathrm{~min}$, is a reliable test for screening non- $C$. albicans and non- $C$. tropicalis yeasts.
\end{abstract}

\section{Introduction}

Candidosis caused by several species of the genus Candida is the most widespread and prevalent mycotic disease of man. ${ }^{1}$ It may be superficial or a systemic infection; superficial infections are the most common. Systemic candidosis is a serious problem, predominantly of temporarily or chronically compromised hosts, and affects the internal organs of vital importance. ${ }^{2}$ The most important causative organisms are $C$. albicans and C. tropicalis as they are responsible for most of the superficial and systemic mycoses..$^{3,4}$

Most of the biochemical tests used for the identification of yeasts suffer from the disadvantage that the results are obtained relatively slowly. The use of a rapid assay for the identification of $C$. albicans with 4methylumbelliferyl- $\mathrm{N}$-acetyl- $\beta$-D-galactosaminide was first reported by Perry and Miller, ${ }^{5}$ and was arbitrarily designated as UAG.

The present study was initiated to evaluate the reliability of the 4-methylumbelliferyl substrate in differentiating $C$. albicans, a principal human pathogen, from $C$. tropicalis and other yeasts. Yeast cultures were tested for the ability to produce blue fluorescence with UAG both as primary and as pure cultures.

The UAG test is a biochemical test that relies on detecting pre-formed or newly formed C. albicans $\beta$-Dgalactosaminidase. ${ }^{5}$ This enzyme hydrolyses ÚAG to 4-methylumbelliferone (4-MU) that produces a blue fluorescence at $365 \mathrm{~nm}$.
The identity of the clinical isolates tested with UAG was confirmed by tests for chlamydospore ( $\mathrm{CHL}$ ) and germ-tube (GT) formation for C. albicans and morphology, fermentation and assimilation tests for $C$. tropicalis and other yeasts.

\section{Materials and methods}

\section{UAG test}

UAG (0.15 mM) was dissolved in $1 \mathrm{ml}$ of dimethyl sulphoxide and diluted to $100 \mathrm{ml}$ with acetate buffer, pH 5.1. The substrate was sterilised by filtration with a membrane filter (Millipore Corp., Bedford, MA, USA). It was then dispensed in borosilicate tubes $(7 \times 120 \mathrm{~mm})$ for immediate use or stored at $-4^{\circ} \mathrm{C}$. Borosilicate tubes were used to avoid metal ions interfering with the reaction, which is likely if ordinary test tubes are used. For the same reason the UAG solution was inoculated with a wooden applicator to give a heavy milky suspension and mixed with a vortex mixer. The suspension was incubated at $37^{\circ} \mathrm{C}$ and examined for the presence or absence of blue fluorescence with a Wood's lamp $(365 \mathrm{~nm})$ in a partially darkened room every $30 \mathrm{~min}$ for $2 \mathrm{~h}$. For positive tests, the substrate-yeast mixture produced a blue fluorescence within $30 \mathrm{~min}$; lack of blue fluorescence indicated a negative result.

A positive control strain of $C$. albicans, selected for its ability to produce blue fluorescence with UAG, CHL on rice cream and GTs in both serum and rabbit coagulase plasma, was used. The other controls were an uninoculated tube of substrate and an inoculated tube of solvent without conjugate, to ensure that the 
conjugate had not deteriorated and that the solvent had not caused the fluorescence.

\section{Ox serum and rabbit plasma with tryptic soy broth}

Many laboratories use non-human serum as the GT induction medium ${ }^{6}$ because of safety problems associated with human serum, especially since the recognition of the human immunodeficiency virus (HIV) and the acquired immunodeficiency syndrome (AIDS).

Ox serum $(0.3 \mathrm{ml})$ and Rabbit Coagulase Plasma (RCP; BBL) $(0.3 \mathrm{ml})$, each mixed with $0.2 \mathrm{ml}$ of tryptic soy broth, were investigated for their ability to induce GT. RCP with EDTA was reconstituted with sterile distilled water and used within 14 days. Germ-tube induction medium $(0.5 \mathrm{ml})$ was mixed in a test tube with a yeast grown for 24 or $48 \mathrm{~h}$ on Sabouraud dextrose agar. The suspension was incubated at $37^{\circ} \mathrm{C}$ with a sterile pipette in the test tube which was used to withdraw a drop of yeast suspension to mount on a slide for examination for GT formation. The GT definition used was that of Berardinelli and Opheim. ${ }^{6}$

GT formation was examined at 30-min intervals for $2 \mathrm{~h}$; a minimum of 100 cells was examined by light microscopy at a magnification of $\times 250$.

\section{Identification}

Eighty-two clinical yeast isolates were tested for carbohydrate and nitrogen assimilation profiles and glucose fermentation. The yeasts were identified with the aid of a computer program--"Yeast Identification Program". The carbohydrate and nitrogen assimilation profiles used in the program are: D-glucose fermentation; growth or lack of growth on Dgalactose, D-xylose, maltose, cellobiose, lactose, raffinose, L-arabinitol, myo-inositol, 2-keto-D-gluconate, DL-lactate, citrate, L-arabinose, D-gluconate, nitrate, nitrite, ethylamine, creatinine, rhamnose and cycloheximide $0.01 \%$; growth at $37^{\circ} \mathrm{C}$ or $42^{\circ} \mathrm{C}$, or both; and the presence of pink pigmentation. Additional tests are available on the program in the event that there is no definitive species identification based on the results of the above tests.

\section{Results}

\section{Reaction of C. albicans and C. tropicalis with $U A G$}

Based on three tests-CHL, GT and UAG-the possible result combinations for $C$. albicans and $C$. tropicalis are shown in table I. The theoretically expected results are based on the assumption that the UAG test is positive only with $C$. albicans and always negative with $C$. tropicalis. Therefore, combination no. 1 is theoretically typical of $C$. albicans and combination no. 6 of $C$. tropicalis. The other combinations-nos. 2, 3 and 4 -are theoretically possible for $C$. albicans while no. 5 identifies $C$. tropicalis. The results of CHL, GT and UAG tests
Table I. Theoretically expected result combinations based on chlamydospore (CHL) production, germ-tube (GT) formation and the UAG reaction

\begin{tabular}{cccc}
\hline Combination no. & CHL & GT & UAG \\
\hline 1 & + & + & + \\
2 & - & + & + \\
3 & + & - & + \\
4 & + & + & - \\
5 & - & - & + \\
6 & - & - & -
\end{tabular}

Combination no. 1 is typical for $C$. albicans; no. 6 is typical for $C$. tropicalis; nos. 2, 3 and 4 are theoretically possible for atypical $C$. albicans; no. 5 is theoretically possible for atypical $C$. tropicalis.

Table II. Experimental results obtained with $C$. albicans and C. tropicalis strains

\begin{tabular}{ccccccc}
$\begin{array}{c}\text { Combination } \\
\text { no. }\end{array}$ & CHL & GT & UAG & $\begin{array}{c}\text { Number } \\
\text { of strains }\end{array}$ & Identification \\
\hline 1 & + & + & + & 48 & typical $C$. albicans \\
6 & - & - & - & 11 & typical $C$. tropicalis \\
5 & - & - & + & 18 & atypical $C$. tropicalis \\
2 & - & + & + & 5 & atypical $C$. albicans \\
\hline
\end{tabular}

Table III. UAG reactions in typical $C$. albicans and $C$. tropicalis strains and atypical $C$. albicans strains

\begin{tabular}{crrrrrr}
\hline & \multicolumn{5}{c}{ Number of strains } \\
\cline { 2 - 6 } $\begin{array}{c}\text { Combination } \\
\text { no.* }\end{array}$ & GT + & GT - & \multicolumn{4}{c}{ UAG colour intensity $\dagger$} \\
\cline { 2 - 7 } & & & 3 & 2 & 1 & 0 \\
\hline 1 & 48 & 0 & 43 & 5 & 0 & 0 \\
6 & 0 & 11 & 0 & 0 & 0 & 11 \\
5 & 0 & 18 & 14 & 2 & 2 & 0 \\
2 & 5 & 0 & 5 & 0 & 0 & 0 \\
Total & 53 & 29 & 62 & 7 & 2 & 11
\end{tabular}

*1, typical $C$. albicans; 6 , typical $C$. tropicalis; 2 , atypical $C$. albicans; 5 , atypical $C$. tropicalis. Only typical $C$. albicans strains formed chlamydospores, while the other yeast isolates were all negative for chlamydospores.

$\nmid 3$, intensity of fluorescence as in positive control; 2, less fluorescence compared to control; 1, much less fluorescence compared to 2 and control; 0 , no fluorescence.

were used together with the morphology, assimilation and fermentation tests to provide a complete identification profile.

The result combinations nos. 3 and 4 were not obtained. As can be seen in table II, 48 isolates gave results typical for C.albicans and 11 for C.tropicalis. This represents $72 \%$ (59) typical strains. Of the remaining strains, none was able to form chlamydospores except for the five isolates that formed a germ tube and were UAG positive. The other 18 isolates were UAG positive only but gave negative results in CHL and GT tests. Two-thirds of the results obtained in this study were those theoretically expected.

Based on the morphology, fermentation and assimilation results, the 18 strains listed in tables II and III were considered to be $C$. tropicalis, although they gave positive UAG results, while five strains con- 
Table IV. Results of CHL, GT and UAG reaction for yeasts other than $C$. albicans and $C$. tropicalis

\begin{tabular}{|c|c|c|c|c|c|c|c|}
\hline \multirow{3}{*}{$\begin{array}{l}\text { Yeast } \\
\text { species }\end{array}$} & \multicolumn{7}{|c|}{ Number of strains } \\
\hline & \multirow{2}{*}{$\begin{array}{c}\mathrm{CHL} \\
\text { negative }\end{array}$} & \multirow{2}{*}{$\begin{array}{c}\text { GT } \\
\text { negative }\end{array}$} & \multicolumn{4}{|c|}{$\begin{array}{l}\text { UAG colour } \\
\text { intensity }\end{array}$} & \multirow{2}{*}{ Total } \\
\hline & & & 3 & 2 & 1 & 0 & \\
\hline C. parapsilosis & 30 & 30 & 0 & 0 & 0 & 30 & 30 \\
\hline D. hansenii & 19 & 19 & 0 & 0 & 2 & 17 & 19 \\
\hline Cr. neoformans & 17 & 17 & 0 & 0 & 2 & 15 & 17 \\
\hline C. guilliermondii & 14 & 14 & 0 & 0 & 0 & 14 & 14 \\
\hline C. glabrata & 3 & 3 & 0 & 0 & 0 & 3 & 3 \\
\hline S. pombe & 2 & 2 & 0 & 0 & 0 & 2 & 2 \\
\hline C. lusitaniae & 2 & 2 & 0 & 0 & 0 & 2 & 2 \\
\hline$T$. beigelii & 9 & 9 & 1 & 0 & 3 & 5 & 9 \\
\hline$G$. candidum & 2 & 2 & 0 & 0 & 0 & 2 & 2 \\
\hline$T$. candida & 2 & 2 & 0 & 0 & 0 & 2 & 2 \\
\hline C. kefyr & 1 & 1 & 0 & 0 & 0 & 1 & 1 \\
\hline Rh. minuta & 1 & 1 & 0 & 0 & 0 & 1 & 1 \\
\hline Cr. lutoelus & 1 & 1 & 0 & 0 & 0 & 1 & 1 \\
\hline S. cerevisiae & 1 & 1 & 0 & 0 & 0 & 1 & 1 \\
\hline Cr. lipolytica & 1 & 1 & 0 & 0 & 0 & 1 & 1 \\
\hline Cr. rugosa & 1 & 1 & 0 & 0 & 0 & 1 & 1 \\
\hline Total & 106 & 106 & 1 & 0 & 7 & 98 & 106 \\
\hline
\end{tabular}

For UAG colour intensities see table III. C, Candida; $\mathrm{Cr}$, Cryptococcus; S, Saccharomyces; G, Geotrichum; T, Trichosporon; $\mathrm{Rh}$, Rhodotorula: D, Debaryomyces.

sidered to be $C$. albicans were unable to form chlamydospores. These results suggest that at least $10 \%$ of $C$. albicans isolates may fail to form chlamydospores. However, fermentation and assimilation pattern results, as analysed with the "Yeast Identification Program"," confirmed that the five isolates can be considered to be $C$. albicans and the 18 isolates can be considered to be $C$. tropicalis strains that gave "false" positive UAG results. Although the UAG reaction could be read within $30 \mathrm{~min}$, it was monitored for $2 \mathrm{~h}$ to ensure that there was no further colour change to that observed after $30 \mathrm{~min}$.

\section{Reaction of other yeasts with $U A G$}

The reactions of other yeasts (non-C. albicans, nonC. tropicalis) tested with UAG were more reliable. Only one isolate of Trichosporon beigelii gave a positive blue fluorescence with UAG similar to the positive control. The other three isolates of $T$. beigelii, and two isolates each of Debaryomyces hansenii and Cryptococcus neoformans gave only weakly positive reactions (table IV).

\section{Discussion}

In recent years, a significant increase in the number of yeast infections has been reported. ${ }^{4}$ Most infections

\section{References}

1. Odds FC. Candida and candidosis, 2nd edn. London, Bailliere Tindall. 1988

2. Caugant DA, Sandven P. Epidemiological analysis of Candida albicans strains by multilocus enzyme electrophoresis. $J$ Clin Microbiol 1993; 31 : 215-220. are attributed to $C$. albicans. However, other yeast species have been implicated, such as $C$. tropicalis. ${ }^{1,8}$ C. albicans and $C$. tropicalis are found mostly as the cause of systemic mycoses, especially with the increasing use of antibiotics and immunosuppressive chemotherapy. AIDS has further added to the numbers of immunosuppressed hosts, leading to a rise in the number of cases of candidosis and cryptococcosis.

The differentiation of C. albicans from C. tropicalis by traditional methods is still problematic because of the formation of germ-tube-like structures by some strains of $C$. tropicalis. $^{9}$ The isolates used in this study were from samples of sputum, vaginal swabs, pleural puncture, dental protheses, skin scrapings and stomatitis. A similar selection of isolates was used by Hellstein et al.$^{10}$ where $C$. albicans from diseased and non-diseased hosts gain differences in biochemical properties and colony phenotypic characteristics. A particular pattern of characteristics may predict the behaviour of candidal isolates in disease.

The reasons for the inability of the five isolates of $C$. albicans to form chlamydospores are not clear; it may be due to the choice of medium, too heavy an inoculum or to some other inhibitory factors. For example, Young et al. ${ }^{11}$ reported the inhibition of $C$. albicans growth in the $\mathrm{pH}$ range $3 \cdot 3-4 \cdot 5$, especially when the $\mathrm{pH}$ was adjusted by adding lactic acid. However, if the $\mathrm{pH}$ was adjusted with tartaric acid, inhibition was less. Assimilation of nutrients, especially the utilisation of organic acids, is affected by $\mathrm{pH}$. The five isolates above were tested three separate times on rice cream agar, but no chlamydospores were formed. The possibility of non-chlamydospore-forming C. albicans isolates should not be discounted.

When examining a yeast for chlamydospores, especially with $C$. tropicalis, careful observation is essential to differentiate chlamydospore-like structures from the true chlamydospores of $C$. albicans. To induce germ-tube structures, media containing nonhuman serum are useful. In this study, ox serum was superior to RCP in tryptic soy broth for inducing germ tubes with $C$. albicans. Some isolates of $C r$. neoformans var. gattii of serotype $\mathrm{C}$ were able to form germ-tubelike structures. Other studies have also reported formation of germ-tube-like structures in other yeasts, such as C. tropicalis, C. parapsilosis and Cr. gastricus. ${ }^{5}$ Therefore, discrepant structures resembling germ tubes must be distinguished.

I thank Janssen Pharmaceutica and Gamma Pharmaceuticals Ltd for financial support, Professor C. De Vroey and Dr D. Swinne for their suggestions, C. Raes-Wuytuck for technical support and Professor D. Morgan for encouragement. Microbiol Rev 1990; 3: 32-45.

5. Perry JL, Miller GR. Umbelliferyl-labeled galactosaminide as an aid in identification of Candida albicans. J Clin Microbiol 1987; 25 : 2424-2425. 
6. Berardinelli S, Opheim DJ. New germ tube induction medium for the identification of Candida albicans. J Clin Microbiol 1985; 22: 861-862.

7. De Vroey C, Raes-Wuytuck C. Yeast identification program. Antwerp, Belgium, Medical and Veterinary Mycology Laboratory. 1988.

8. Koneman EW, Roberts GD. Practical laboratory mycology, 3rd edn. London, Williams and Wilkins. 1985: 143.
9. Pfaller MA. Laboratory aids in the diagnosis of invasive candidiasis. Mycopathologia 1992; 120: 65-72.

10. Hellstein JH, Fotos PG, Law SS, Kovacevic M, Carriere KC Differentiation of sugar assimilation characteristics and colony phenotypes in pathogenic and commensal oral candidal isolates. J Oral Pathol Med 1993; 22: 312-319.

11. Young G, Krasner RI, Yudkofsky PL. Interactions of oral strains of Candida albicans and Lactobacilli. J Bacteriol $1956 ; 72: 525-529$. 